\title{
A Comparison of Subxiphoid and Intercostal Wound Pain in the Same Patients Following Thoracic Surgery
}

\section{Yu-Wei Liu ( $\nabla$ nipma6714@gmail.com )}

Kaohsiung Medical University Hospital, Kaohsiung Medical University

Shah-Hwa Chou

Kaohsiung Medical University Hospital, Kaohsiung Medical University

Andre Chou

Poznań University of Medical Sciences

Chieh-Ni Kao

Kaohsiung Medical University Hospital, Kaohsiung Medical University

Hung-Hsing Chiang

Kaohsiung Medical University Hospital, Kaohsiung Medical University

Jui-Ying Lee

Kaohsiung Medical University Hospital, Kaohsiung Medical University

Hsien-Pin Li

Kaohsiung Medical University Hospital, Kaohsiung Medical University

Po-Chih Chang

Kaohsiung Medical University Hospital, Kaohsiung Medical University

\section{Research Article}

Keywords: subxiphoid, intercostal, VATS, postoperative pain

Posted Date: September 1st, 2021

DOl: https://doi.org/10.21203/rs.3.rs-846594/v1

License: (c) (i) This work is licensed under a Creative Commons Attribution 4.0 International License. Read Full License 


\section{Abstract}

Background: There is a lack of data comparing postoperative pain after subxiphoid and intercostal video-assisted thoracoscopic surgery (VATS). Pain is an individual's subjective experience and therefore difficult to compare between different individuals subjected to either procedure. This study assessed reported pain at six postoperative time points in the same patients receiving both subxiphoid and intercostal incisions for thoracic disease.

Methods: Data from 26 patients who received simultaneous combined intercostal and subxiphoid VATS were retrospectively analyzed from August 2019 to April 2020. All patients received same length subxiphoid and intercostal incisions with or without drain placements. A numerical pain rating scale was administered on postoperative days (POD)-1, POD-2, POD-Discharge, POD30, POD-90, and POD-180.

Results: Bilateral uniportal VATS was performed in 11 patients and unilateral multiportal VATS in 15 patients. In the unilateral VATS group, there were no significant differences in pain reported for subxiphoid and intercostal incisions. However, in the bilateral VATS group, subxiphoid wounds resulted in significantly higher mean pain scores on POD-1, POD-2, and PODDischarge $(p=0.0003,0.001$, and 0.03 , respectively) but lower pain scores on POD-90 $(p=0.03)$.

Conclusions: In the bilateral VATS group, higher late (3 and 6 months) postoperative pain was associated with intercostal incisions as have been reported by previous studies. Interestingly, subxiphoid incisions were found to have higher early (Day 1 , 2 , and Discharge) postoperative pain than intercostal incisions. More research is needed to better understand the cause of pain in a surgical area previously not thought to be prone to pain.

\section{Introduction}

Recent data comparing open thoracotomy to video-assisted thoracoscopic surgery (VATS) suggests a predilection to the latter. Randomized and non-randomized studies have associated VATS procedures with less trauma, decreased postoperative pain, fewer postoperative complications and faster recovery. Findings such as these has seen a wider usage among thoracic surgeon [1-3]. Continued advances in minimally invasive thoracic surgery have permitted the evolution from 3-or 4-port VATS techniques to the uniportal technique. Uniportal VATS is becoming more popular globally due to the smaller number of incisions and decreased postoperative chest wall neuralgia, and, as suggested by one study, more ergonomic than other multiport techniques [4].

It has been suggested that uniportal VATS is less painful than multiportal VATS based on the premise that one incision is less painful than two or more. However, questions have arisen regarding the relative degree of postoperative pain and chest wall neuralgia among patients receiving uniportal VATS compared with those who received multiportal VATS [5-8]. In principle, VATS is a minimal access surgical technique, but as much as one-third of patients receiving these procedures report chronic pain (pain lasting 2 to 3 months) possibly due to intercostal nerve compression [9]. It is reasonable then that subxiphoid uniportal VATS, an approach which avoids the intercostal spaces, should reduce postoperative pain.

In 2012, Suda et al. reported their first concomitant use of subxiphoid single-port thymectomy and $\mathrm{CO}_{2}$ insufflation [10]. Not long after in 2014, Liu et al. reported their first use of subxiphoid uniportal VATS lobectomy [11], followed by subsequent use of this alternative approach to treat a wide array of thoracic procedures worldwide [12-16]. This relatively new technique often requires division of the rectus abdominis muscle, resection of the xiphoid process and use of a sternal elevator. However, these added manipulations could potentially offset any pain advantages gained from not injuring the intercostal nerves [17]. Moreover, proponents of its use have admitted that there may be some cardiac compression when using subxiphoid VATS to perform left thoracic procedures as well as limited visualization of the posterior mediastinum and potential technical difficulties [11-16].

While theoretically there should be less pain, is there actually less pain? Pain is a subjective and multidimensional experience. One of the most challenging aspects of any pain research is the objective evaluation of pain since pain is an individual experience. Although several studies have been performed on the incidence and intensity of pain after thoracic surgery $[9,18-$ 
23], to the best of our knowledge, none have used the same patients to investigate postoperative pain associated with both subxiphoid and intercostal incisions. A comparison of the postoperative pain associated with the two approaches in the same patients should reduce some bias arising from comparing use of one approach on one group of patients with the use of the other approach on a different group of patients.

Therefore, this study adopted both subxiphoid and intercostal incisions with the same lengths on the same thoracic disease patients. A numerical pain rating scale was analyzed at six different postoperative time points, three early and three late.

\section{Materials And Methods Study design}

The study was approved by the research ethics committee and waived the requirement for written informed consent at Kaohsiung Medical University Hospital (Approval number: KMUHIRB-E(I)-20200228). 30 patients were consecutively enrolled receiving combined subxiphoid and intercostal VATS for thoracic procedures at a single medical center from August 2019 to April 2020. All incisions were equal in length. None of the patients had preoperative analgesic requirements, previous thoracic surgeries, impaired cardiac functioning, or body mass indices $(\mathrm{BMI})>30 \mathrm{~kg} / \mathrm{m}^{2}$. Two patients were excluded whose subxiphoid and intercostal incisions were not the same length, one whose postoperative drains were not the same size, and one who received intraoperative thoracic epidural anesthesia. After exclusion, 26 patients were left divided into two groups, (1) eleven patients receiving simultaneous bilateral uniportal VATS $(n=11)$ with ipsilateral subxiphoid incisions on one side and contralateral intercostal incisions on the other and (2) fifteen patients receiving unilateral multiportal VATS $(n=15)$ with both surgical approaches used on the same side (Fig. 1). All procedures were performed by one surgical team following the same perioperative protocols. Patient data was collected including age, gender, BMI, smoking habits, lung function tests, perioperative data, postoperative complications, and follow-up pain scores from electronic medical records. All methods were carried out in accordance with relevant guidelines and regulations.

\section{Operative technique}

\section{Simultaneous bilateral uniportal VATS}

Under general anesthesia via a double-lumen endotracheal tube, nine of the simultaneous bilateral uniportal VATS patients received right side subxiphoid VATS pulmonary wedge resections and left side intercostal VATS pulmonary wedge resections. The other two patients received right side intercostal VATS upper lobectomies and left side subxiphoid VATS lower lobe wedge resections (See Supplemental Fig. 1 and Supplemental table 1). Surgical equipment included Echelon Flex endoscopic articulating linear cutters (Ethicon Endo-Surgery, Somerville, NJ, USA), long curved dissector and grasping forceps with double articulations, and curved suction (Scalan International, Inc. Saint Paul, Minnesota, USA), and a 10-mm 30 thoracoscope (Karl Storz, Munich, Germany). Patients were placed in a supine position on a tilted operating table (30 to 45 degrees) for easy access and performance of subxiphoid VATS surgery. A single incision 2-5 cm long was made below the xiphoid process and slightly slanted along the costal arch. The skin, subcutaneous tissue, and rectus abdominis were cut, blunt dissection was performed using a finger applied to the deep surface of the costal arch, and the pleural cavity was accessed. A plastic wound protector (Alexis ${ }^{\circledR}$, Applied Medical, California, USA) was placed in the incision. Throughout the procedure, the xiphoid process was not excised and no sternal elevator was used. After uniportal subxiphoid VATS pulmonary wedge resection, a chest drain was inserted through the same incision. For the other side, the patient was changed to a decubitus position access the contralateral lung. A single incision with the same length as the subxiphoid incision was made over the intercostal space and a wound protector inserted. A chest drain the same size as used on the other side was placed through the intercostal incision (Fig. 2a).

\section{Unilateral multiportal VATS}

Following similar anesthetic procedures and positioning for the optimized subxiphoid approach, multiportal combined subxiphoid and intercostal VATS was performed depending on surgical procedure type (See Supplemental Fig. 2 and Supplemental table 2). Incisions equal in length were made over the subxiphoid and intercostal regions. For patients with 
pulmonary or mediastinal tumors, specimens were retrieved through the subxiphoid incision without enlarging the wound. Nine of the 15 patients received an additional small third port $(0.5-1 \mathrm{~cm})$ for intraoperative manipulation and placement of postoperative chest drains (Fig. 2b). The other six patients received 2-port VATS, some patients $(n=2)$ receiving two equal but separate drains via the subxiphoid and intercostal incisions and others $(n=4)$ receiving no chest drain.

\section{Postoperative management}

Chest radiography was performed immediately after the operation or the following morning. Chest drains were removed in both groups if there were no air leaks and if the drainage was $<200 \mathrm{ml}$ within 24 hours. Fifteen of the 26 patients received intravenous patient-controlled anesthesia upon request. Acetaminophen, diclofenac, and tramadol were regularly administered once patients resumed normal oral intake until discharge. Additional doses of intravenous Parecoxib were used for intolerable pain during postoperative hospital stay. Pain scores for both subxiphoid and intercostal wounds were simultaneously assessed separately by a surgical team member using a numerical rating scale (NRS), 0 (no pain) to 10 (excruciating pain), every eight hours with patient at rest on postoperative day-1 (POD-1) and then on subsequent postoperative days until discharge. The mean of the three daily NRS scores for both incisions were recorded for the three early time points. Pain scores at the three late time points were followed up either by a consultation in the outpatient clinic or a phone call.

\section{Statistical Analysis}

Descriptive data were expressed as numbers with percentages and continuous variables expressed as either means with standard deviation or medians with interquartile range (IQR). The Student's $t$ test (paired t-test) was used to compare subxiphoid and intercostal NRS scores from time point to time point among the same patients. All statistical operations were performed using SPSS 20 (SPSS Inc., Chicago, IL, USA) for Windows. All statistical tests were two-tailed and $P$-value $<0.05$ was considered significant.

\section{Results}

In this review of the data collected from 26 patients receiving subxiphoid VATS for the surgical treatment of thoracic conditions between August 2019 and April 2020, eleven simultaneously received uniportal ipsilateral subxiphoid VATS on one side and contralateral intercostal VATS on the other side, while fifteen received multiportal subxiphoid and intercostal VATS on one side only. As can be seen in Table 1, males made up sixty-one percent and mean BMI was $23 \mathrm{~kg} / \mathrm{m}^{2}$. Operative time, blood loss, complications, and postoperative hospital length-of-stay were comparable and similar to those reported in the subxiphoid VATS literature [12-16]. 
Table 1

Characteristics of enrolled patients $(n=26)$

\begin{tabular}{|ll|}
\hline Characteristic & Value \\
\hline Mean age (range), y & $52(18-76)$ \\
\hline Gender, \% (n) & $61(16)$ \\
\hline Male & $39(10)$ \\
\hline Female & $42(11)$ \\
\hline Smoking (yes), \% (n) & $23(16-30)$ \\
\hline Mean BMI (range), kg/m² & \\
\hline Pulmonary function test & $2.4(1.4-3.6)$ \\
\hline Mean FEV1 (range), L & $85(58-115)$ \\
\hline Mean FEV1 (range), Predicted \% & $16(4)$ \\
\hline Grade I-Il complication, \% (n) & $8(2)$ \\
\hline Prolonged air leak (> 5 days) & $4(1)$ \\
\hline Atrial fibrillation & $4(1)$ \\
\hline Wound allergy & $4(2-9)$ \\
\hline Mean postoperative stay (range), day & $3.0(2.0-5.0)$ \\
\hline Median wound length (range), cm & $16(12-28)$ \\
\hline Median drain size (range), Fr & $100(40-220)$ \\
\hline Median operation time (range), min & $10(5-200)$ \\
\hline Median blood loss (range), ml & (n) body mass index; FEV1: forced expiratory volume in the first second of expiration \\
\hline BMI: bo & \\
\hline
\end{tabular}

As can be seen in Supplemental table 1, out of the eleven patients receiving bilateral VATS, five had primary spontaneous pneumothorax with contralateral blebs for which they received surgical treatments following previous reported procedures [24] and six had indeterminate lung nodules or suspected lung cancer and received simultaneous bilateral operations for accurate diagnosis and staging after weighing relative risks and benefits. Of the fifteen patients who received unilateral VATS, two lung cancer patients who had malignant pericardial effusion received pericardial windows and the remaining 13 had pulmonary or mediastinal tumors (See Supplemental table 2). Based on our previous experience, postoperative drains were not placed in patients following mediastinal tumor resection [25]. For patients who had received pulmonary lobectomies and wedge resections and who were at risk of air leaks, chest drains were positioned from a small third port incision. In both groups, all subxiphoid and intercostal incisions and drains, when created, were equal in incision length and drain size (Figs. 3 and 4 ).

Table 2 shows the mean subxiphoid incision and intercostal incision NRS pain score results for three postoperative hospital days, Post-op Day 1, 2, and Discharge and three postoperative follow-up days 30, 90, and 180. In patients receiving bilateral VATS (on two sides), subxiphoid incisions had significantly higher mean pain scores than the intercostal incisions in the early postoperative period (POD-1, POD-2, and POD-Discharge $[p=0.0003,0.001$, and 0.03 , respectively]) (Fig. 5) but lower mean pain scores on POD-90 and POD-180 ( $p=0.03$ and 0.16$)$. Interestingly, in patients receiving unilateral VATS, the pain score differences between the two incisions were insignificant regardless of postoperative assessment time (All $p>0.05)($ Fig. 6). 
Table 2

Postoperative numerical rating scale (NRS) pain score

\begin{tabular}{|c|c|c|c|c|c|c|c|c|c|c|}
\hline \multirow{2}{*}{$\begin{array}{l}\text { Pain } \\
\text { score }\end{array}$} & \multicolumn{5}{|c|}{ Bilateral VATS $(n=11)$} & \multicolumn{5}{|c|}{ Unilateral VATS $(n=15)$} \\
\hline & $\begin{array}{l}\text { Subxiphoid } \\
\text { wound }\end{array}$ & $\begin{array}{l}95 \% \\
\mathrm{Cl}\end{array}$ & $\begin{array}{l}\text { Intercostal } \\
\text { wound }\end{array}$ & $\begin{array}{l}95 \% \\
\mathrm{Cl}\end{array}$ & $\begin{array}{l}P \\
\text { value }\end{array}$ & $\begin{array}{l}\text { Subxiphoid } \\
\text { wound }\end{array}$ & $\begin{array}{l}95 \% \\
\mathrm{Cl}\end{array}$ & $\begin{array}{l}\text { Intercostal } \\
\text { wound }\end{array}$ & $\begin{array}{l}95 \% \\
\mathrm{Cl}\end{array}$ & $\begin{array}{l}P \\
\text { value }\end{array}$ \\
\hline POD-1 & $5.1 \pm 1.4$ & $\begin{array}{l}(4.1- \\
6.0)\end{array}$ & $2.5 \pm 1.4$ & $\begin{array}{l}(1.5- \\
3.4)\end{array}$ & 0.0003 & $2.2 \pm 1.8$ & $\begin{array}{l}(1.2- \\
3.2)\end{array}$ & $2.3 \pm 1.3$ & $\begin{array}{l}(1.6- \\
3.1)\end{array}$ & 0.75 \\
\hline POD-2 & $3.6 \pm 1.7$ & $\begin{array}{l}(2.5- \\
4.8)\end{array}$ & $1.6 \pm 0.8$ & $\begin{array}{l}(1.1- \\
2.2)\end{array}$ & 0.001 & $1.8 \pm 1.0$ & $\begin{array}{l}(1.2- \\
2.4)^{-}\end{array}$ & $1.7 \pm 1.4$ & $\begin{array}{l}(0.9- \\
2.5)\end{array}$ & 0.85 \\
\hline $\begin{array}{l}\text { POD- } \\
\text { Discharge }\end{array}$ & $1.9 \pm 1.4$ & $\begin{array}{l}(1.0- \\
2.8)\end{array}$ & $1.1 \pm 0.5$ & $\begin{array}{l}(0.7- \\
1.5)\end{array}$ & 0.03 & $0.9 \pm 0.7$ & $\begin{array}{l}(0.5- \\
1.3)\end{array}$ & $1.2 \pm 1.1$ & $\begin{array}{l}(0.6- \\
1.8)\end{array}$ & 0.21 \\
\hline POD-30 & $0.4 \pm 0.9$ & $\begin{array}{l}(-0.3- \\
1.0)\end{array}$ & $0.6 \pm 0.9$ & $\begin{array}{l}(0.0- \\
1.3)\end{array}$ & 0.49 & $0.5 \pm 1.1$ & $\begin{array}{l}(-0.1- \\
1.1)\end{array}$ & $0.7 \pm 1.6$ & $\begin{array}{l}(-0.1- \\
1.6)\end{array}$ & 0.42 \\
\hline POD-90 & $0 \pm 0$ & $\begin{array}{l}(0.0- \\
0.0)\end{array}$ & $0.4 \pm 0.5$ & $\begin{array}{l}(0.0- \\
0.7)\end{array}$ & 0.03 & $0.1 \pm 0.5$ & $\begin{array}{l}(-0.2- \\
0.4)\end{array}$ & $0.3 \pm 0.6$ & $\begin{array}{l}(-0.1- \\
0.6)\end{array}$ & 0.16 \\
\hline POD-180 & $0 \pm 0$ & $\begin{array}{l}(0.0- \\
0.0)\end{array}$ & $0.2 \pm 0.4$ & $\begin{array}{l}(-0.1- \\
0.5)\end{array}$ & 0.16 & $0 \pm 0$ & $\begin{array}{l}(0.0- \\
0.0)\end{array}$ & $0.1 \pm 0.4$ & $\begin{array}{l}(-0.1- \\
0.3)\end{array}$ & 0.16 \\
\hline
\end{tabular}

\section{Discussion}

Pain is usually assessed subjectively by means of verbal or visual intensity scales and questionnaires [9, 21]. Most pain scales are subjective assessment tools because pain is interpreted differently by each individual. In this study, diligent attempts to control as many confounding variables as possible were made - such as the subjective individuality. This was achieved by performing two incisions in the same patients during the same operation session and asking them to rate their incision-related pain at the same six postoperative time points. Incision length, chest tube size and perioperative analgesic protocols were all controlled for. Careful attention toward reducing the influence of these potential confounders should reduce their effect on our results and produce a more reliable comparison of pain following thoracic surgery.

In contrast to the literature's overwhelming opinion that subxiphoid VATS results in much less early postoperative pain than intercostal VATS following thoracic surgery $[12-16,18]$, our study found the subxiphoid incision produced more pain than the intercostal incision during the early postoperative period (POD-1, POD-2, and POD-Discharge), but only in the bilateral VATS group, not in the unilateral VATS group. The authors suggest one reason for the difference may be because the subxiphoid incision was always made below the xiphoid process and slightly tilted in the direction of the operative costal arch. There was greater distance between the two incisions (subxiphoid and intercostal) in the bilateral VATS group than that in the unilateral VATS group, which could possibly decentralize a patient's focus of attention. Another reason may be that nine of 15 patients in the unilateral VATS group received 3-port VATS. The chest drains were placed through a small intercostal port which has been reported to have negative impact on pain in the literature [20]. This would also confound perceptions of pain for other incisions. These two factors could interfere with a clear comparison of pain between subxiphoid and intercostal incisions in the unilateral VATS group.

Although the findings of this study were in contrast to findings in the literature (less early postoperative pain in subxiphoid compared to intercostal VATS), the present study did find higher pain intensity associated with intercostal incisions in the late follow-up period (POD-90 and 180), consistent with the literature [18]. None of the eleven patients receiving bilateral VATS reported chronic pain (pain lasting 2 to 3 months) over the subxiphoid incision, while four (4/11) reported pain over the intercostal incision on POD-90 and two reported pain through until POD-180. One of the fifteen patients receiving unilateral VATS reported pain over both subxiphoid and intercostal incision and two reported pain over the intercostal incisions on POD90. The two patients reporting on POD-90 continued to report the same pain on POD-180. It is interesting to note that five of the 
seven patients who reported chronic pain in our cohort study had primary lung cancers or lung metastases for which they received adjuvant chemotherapy within six months of surgery. One of the seven had pulmonary tuberculosis for which he received six-months of anti-tuberculosis therapy and the other received an unexpected surgical intervention for acute cerebrovascular disorder within three months following thoracic surgery. Previous studies have reported that pain can be expressed or experienced differently depending on race, gender, age, treatment modalities including radiotherapy, pleurectomy, more extensive surgical procedures, and other multifactorial mechanisms [19, 23]. Recently, Yoon and colleagues also reported that adjuvant chemotherapy serves as a risk factor for chronic pain in lung cancer patients after VATS [26], supporting the findings of this study.

The use of subxiphoid VATS incisions avoids intercostal nerve damage, which has often been cited as a major source of postoperative pain and paresthesia after thoracotomy and intercostal VATS. From a surgical standpoint, this approach allows easier access to the anterior mediastinum for thymectomy as well as better access to both sides of the chest with only a single incision. However, this approach, particularly when there is posterior mediastinum and left thorax involvement, can be challenging even for experienced thoracic surgeons. Whether the potential advantages outweigh the potential disadvantages remains to be determined by future studies.

Reviewing the literature, we found it took a long time for the field to draw a firm conclusion regarding relative reported pain associated with thoracotomy versus VATS $[1-3,9,19]$. The debate regarding the relative pain associated with multiportal VATS versus uniportal VATS is also ongoing [5-8]. Until now, only a few studies have reported a reduction in acute postoperative pain and chronic pain associated with subxiphoid VATS $[12-16,18]$.

This study has a number of limitations. The difficulty in recruiting patients who required receiving two procedures in the same locations at the same time meant the limitation of a small sample size was unavoidable. Another limitation is that all the procedures in this study were performed by one surgeon at one institute, and so the learning curve for the performance of subxiphoid VATS has not been taken into consideration. Therefore, further studies including well-controlled prospective studies and multi-center studies are needed to further verify our findings.

\section{Conclusions}

Comparing reported pain associated with two different surgical approaches in each patient, this study found that patients who received simultaneous uniportal ipsilateral subxiphoid VATS and contralateral intercostal VATS reported more pain associated with subxiphoid incision than intercostal incisions. Thus, the use of subxiphoid VATS may not always result in greater pain reduction than intercostal incision in the early postoperative period. Additionally, the higher pain intensity in the intercostal incisions reported in the late postoperative period may not be indicative of pain caused by merely nerve damage during surgery, as there were other potential multifactorial pain-causing or pain-worsening mechanisms such as adjunctive chemotherapy involved.

\section{Declarations}

\section{Acknowledgement:}

The authors thank the help of James Steed on language revision.

\section{Additional Information}

\section{Competing interests:}

All authors declare no competing interests.

\section{Authors' contributions statements:}


Liu YW designed the study. All authors did data acquisition. Liu YW and Chou SH analysed and interpreted the data. All authors wrote the manuscript. All authors read and approved the final manuscript.

\section{Funding and conflict of interest:}

None declared

\section{References}

1. Yan, T.D., Black, D., Bannon, P.G. \& McCaughan, B.C. Systematic review and meta-analysis of randomized and nonrandomized trials on safety and efficacy of video-assisted thoracic surgery lobectomy for early-stage non-small-cell lung cancer. J Clin Oncol. 27, 2553-2562 (2009).

2. Paul, S. et al. Thoracoscopic lobectomy is associated with lower morbidity than open lobectomy: a propensity-matched analysis from the STS database. J Thorac Cardiovasc Surg. 139, 366-378 (2010).

3. Bendixen, M., Jørgensen, O.D., Kronborg, C., Andersen, C. \& Licht, P.B. Postoperative pain and quality of life after lobectomy via video-assisted thoracoscopic surgery or anterolateral thoracotomy for early stage lung cancer: a randomised controlled trial. Lancet Oncol. 17, 836-844 (2016).

4. Rocco, G. One-port (uniportal) video-assisted thoracic surgical resections-a clear advance. J Thorac Cardiovasc Surg. 144, S27-31 (2012).

5. Hirai, K., Takeuchi, S. \& Usuda, J. Single-incision thoracoscopic surgery and conventional video-assisted thoracoscopic surgery: a retrospective comparative study of perioperative clinical outcomest. Eur J Cardiothorac Surg. 49, Suppl 1:i37-41 (2016).

6. Liu, Z., Yang, R. \& Shao, F. Comparison of Postoperative Pain and Recovery between Single-Port and Two-Port Thoracoscopic Lobectomy for Lung Cancer. Thorac Cardiovasc Surg. 67, 142-146 (2019).

7. Tosi, D. et al. Uniportal and three-portal video-assisted thoracic surgery lobectomy: analysis of the Italian video-assisted thoracic surgery group database. Interact Cardiovasc Thorac Surg. 29, 714-721 (2019).

8. Mendogni, P. et al. Uniportal and three-portal video-assisted thoracic surgery pulmonary lobectomy for early-stage lung cancer (UNIT trial): study protocol of a single-center randomized trial. Trials. 22, 163 (2021).

9. Bayman, E.O., Parekh, K.R., Keech, J., Selte, A. \& Brennan, T.J. A Prospective Study of Chronic Pain after Thoracic Surgery. Anesthesiology. 126, 938-951 (2017).

10. Suda, T., Sugimura, H., Tochii, D., Kihara, M. \& Hattori, Y. Single-port thymectomy through an infrasternal approach. Ann Thorac Surg. 93, 334-336 (2012).

11. Liu, C.C., Wang, B.Y., Shih, C.S. \& Liu, Y.H. Subxiphoid single-incision thoracoscopic left upper lobectomy. J Thorac Cardiovasc Surg. 148, 3250-3251 (2014).

12. Wang, B.Y. et al. Thoracoscopic surgery via a single-incision subxiphoid approach is associated with less postoperative pain than single-incision transthoracic or three-incision transthoracic approaches for spontaneous pneumothorax. $J$ Thorac Dis. 8(Suppl 3), S272-278 (2016).

13. Suda, T. et al. Early outcomes in 147 consecutive cases of subxiphoid single-port thymectomy and evaluation of learning curves. Eur J Cardiothorac Surg. 58(Supplement_1), i44-i49 (2020).

14. Yang, X. et al. The Feasibility and Advantages of Subxiphoid Uniportal Video-Assisted Thoracoscopic Surgery in Pulmonary Lobectomy. World J Surg. 43, 1841-1849 (2019).

15. Pfeuty, K. \& Lenot, B. Multiportal subxiphoid thoracoscopic major pulmonary resections. J Thorac Dis. 11, 2778-2787 (2019).

16. Cai, H. et al. Subxiphoid versus intercostal uniportal video-assisted thoracoscopic surgery for bilateral lung resections: a single-institution experience. Eur J Cardiothorac Surg. 57, 343-349 (2020).

17. Licht, P.B. Subxiphoid uniportal lobectomy. Eur J Cardiothorac Surg. 50, 1067 (2016). 
18. Chen, J. et al. Comparison of post-operative pain and quality of life between uniportal subxiphoid and intercostal videoassisted thoracoscopic lobectomy. J Thorac Dis. 12, 3582-3590 (2020).

19. Miyazaki, T. et al. Assessment and follow-up of intercostal nerve damage after video-assisted thoracic surgery. Eur $J$ Cardiothorac Surg. 39, 1033-1039 (2011).

20. Miyazaki, T. et al. Chest tube insertion is one important factor leading to intercostal nerve impairment in thoracic surgery. Gen Thorac Cardiovasc Surg. 62, 58-63 (2014).

21. Maguire, M.F., Ravenscroft, A., Beggs, D. \& Duffy, J.P. A questionnaire study investigating the prevalence of the neuropathic component of chronic pain after thoracic surgery. Eur J Cardiothorac Surg. 29, 800-805 (2006).

22. Maguire, M.F., Latter, J.A., Mahajan, R., Beggs, F.D. \& Duffy, J.P. A study exploring the role of intercostal nerve damage in chronic pain after thoracic surgery. Eur J Cardiothorac Surg. 29, 873-879 (2006).

23. Steegers, M.A., Snik, D.M., Verhagen, A.F., van der Drift, M.A. \& Wilder-Smith, O.H. Only half of the chronic pain after thoracic surgery shows a neuropathic component. J Pain. 10, 955-961 (2008).

24. Liu, Y.W. et al. Simultaneous bilateral thoracoscopic blebs excision reduces contralateral recurrence in patients undergoing operation for ipsilateral primary spontaneous pneumothorax. J Thorac Cardiovasc Surg. 159, 1120-1127.e3 (2020).

25. Liu, Y.W. et al. Is a Chest Tube Necessary after Video-Assisted Thoracoscopic Mediastinal Tumor Resection? Thorac Cardiovasc Surg. 69, 181-188 (2021).

26. Yoon, S. et al. Adjuvant chemotherapy as a risk factor for chronic postoperative pain after video-assisted thoracoscopic surgery: a 10-year single-centre retrospective study. Interact Cardiovasc Thorac Surg. 32, 276-283 (2021).

\section{Figures}

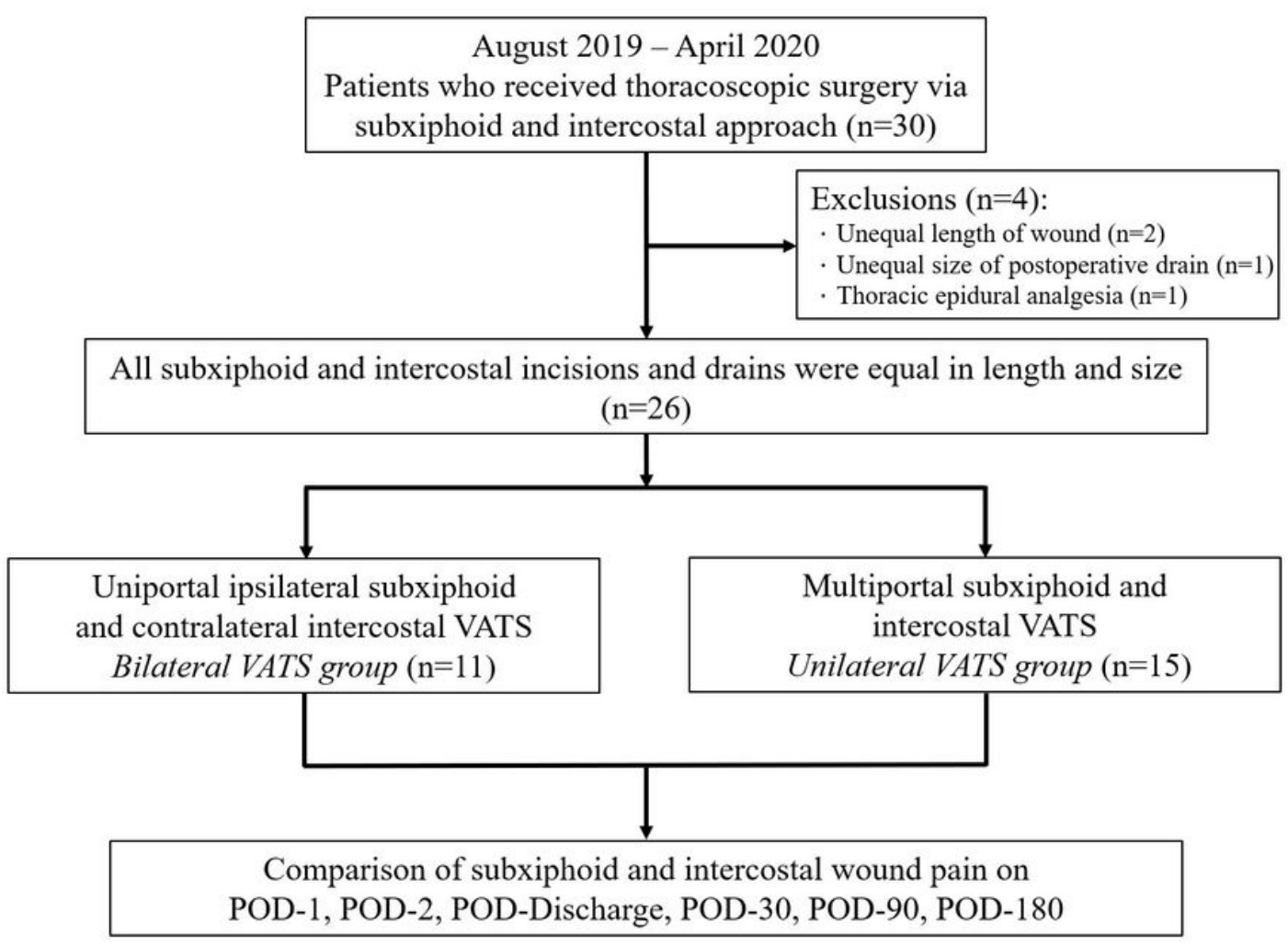

\section{Figure 1}

Flow diagram for patient recruitment VATS, video-assisted thoracoscopic surgery; POD, postoperative day 

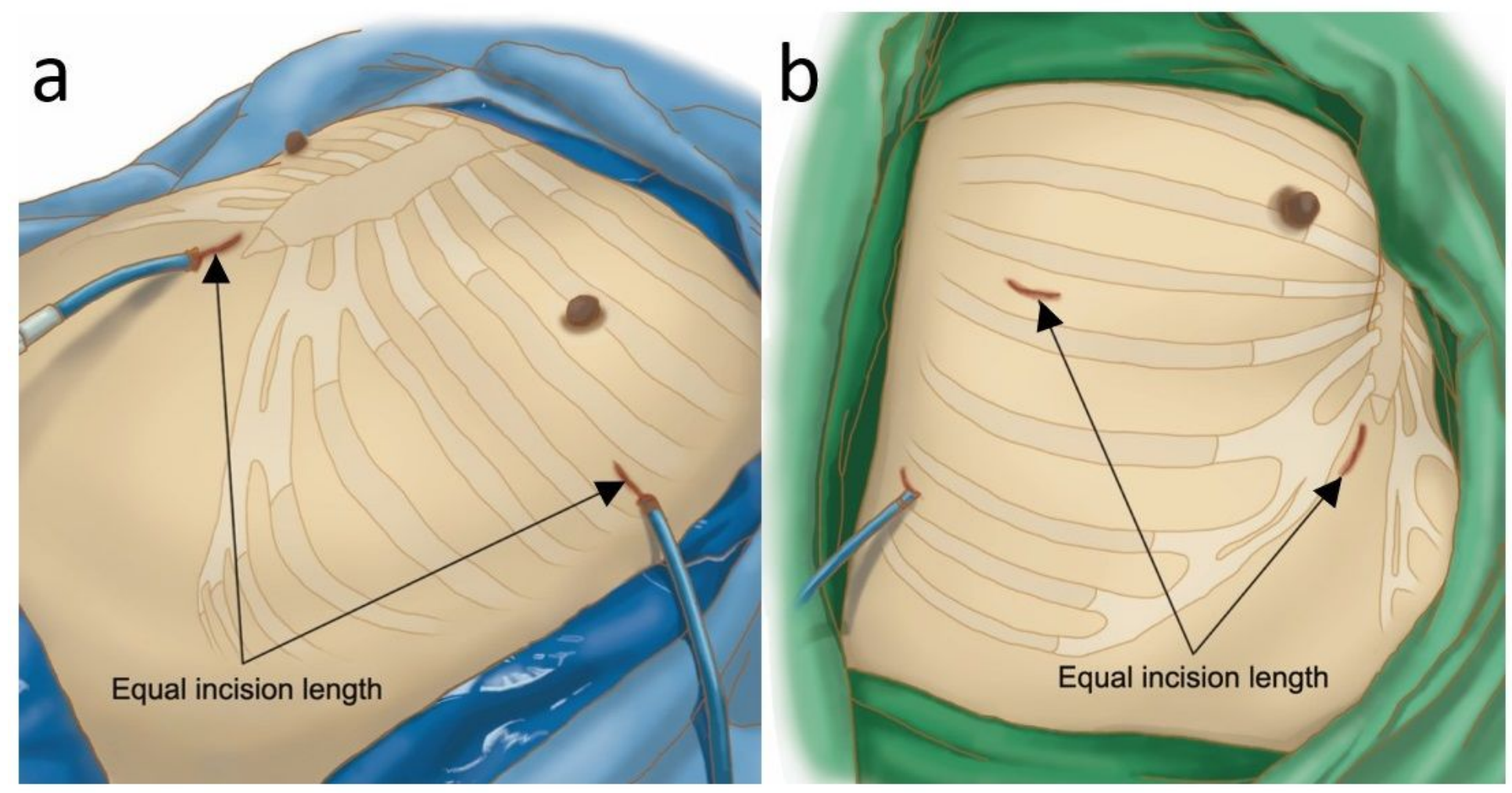

\section{Figure 2}

Postoperative view of subxiphoid and intercostal incision All subxiphoid and intercostal incisions and drains were equal in length and size, either in bilateral uniportal VATS (a) or unilateral multiportal VATS (b). Arrowhead: equal length of subxiphoid and intercostal incisions. VATS, video-assisted thoracoscopic surgery 


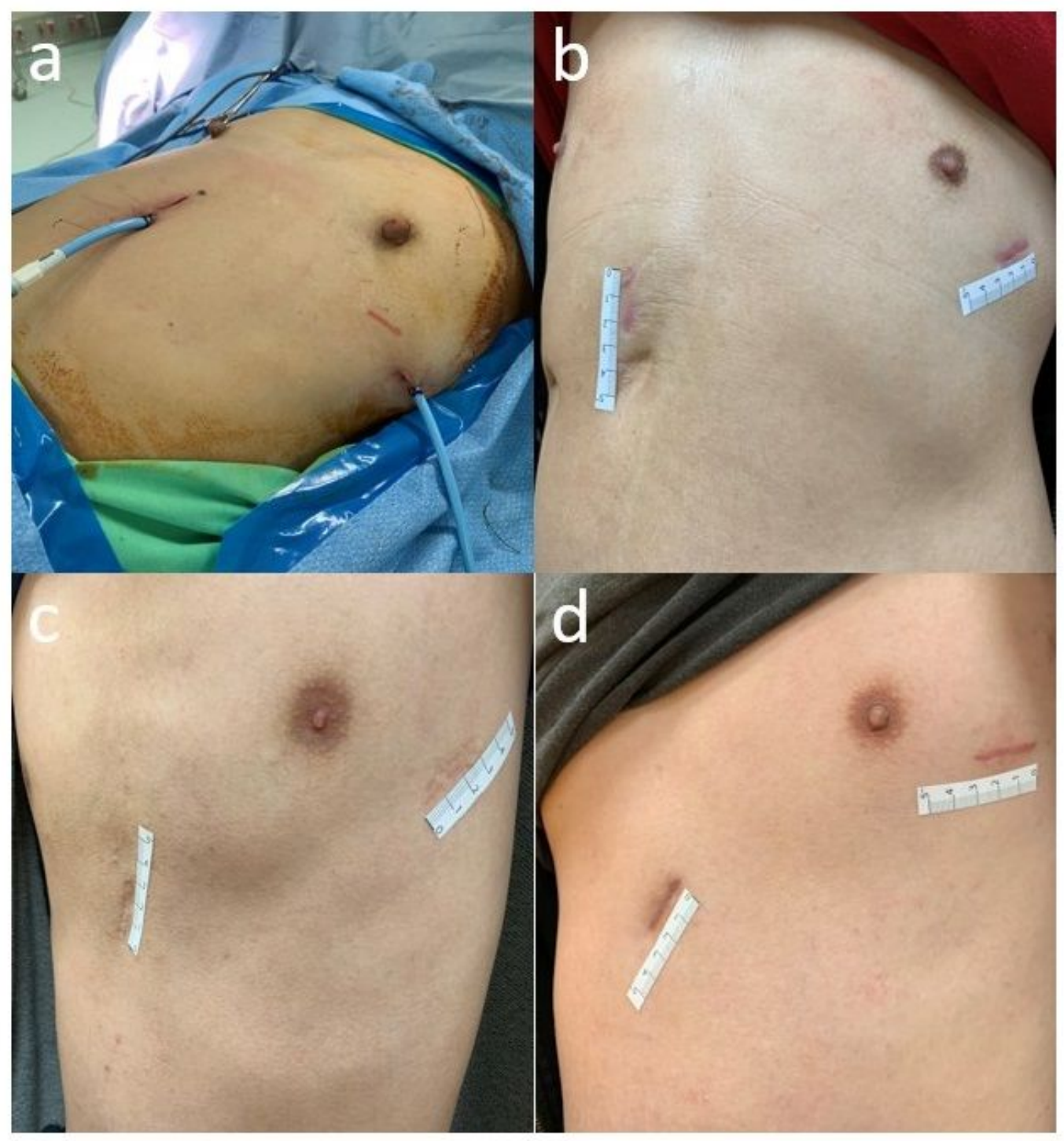

\section{Figure 3}

Patients receiving simultaneous uniportal ipsilateral subxiphoid and contralateral intercostal VATS (a) A patient with equal length of subxiphoid and intercostal incision and drain size. (b) and (c) and (d) Postoperative pictures of three different patients with equal length of subxiphoid and intercostal incisions 3 months following surgery. VATS, video-assisted thoracoscopic surgery 


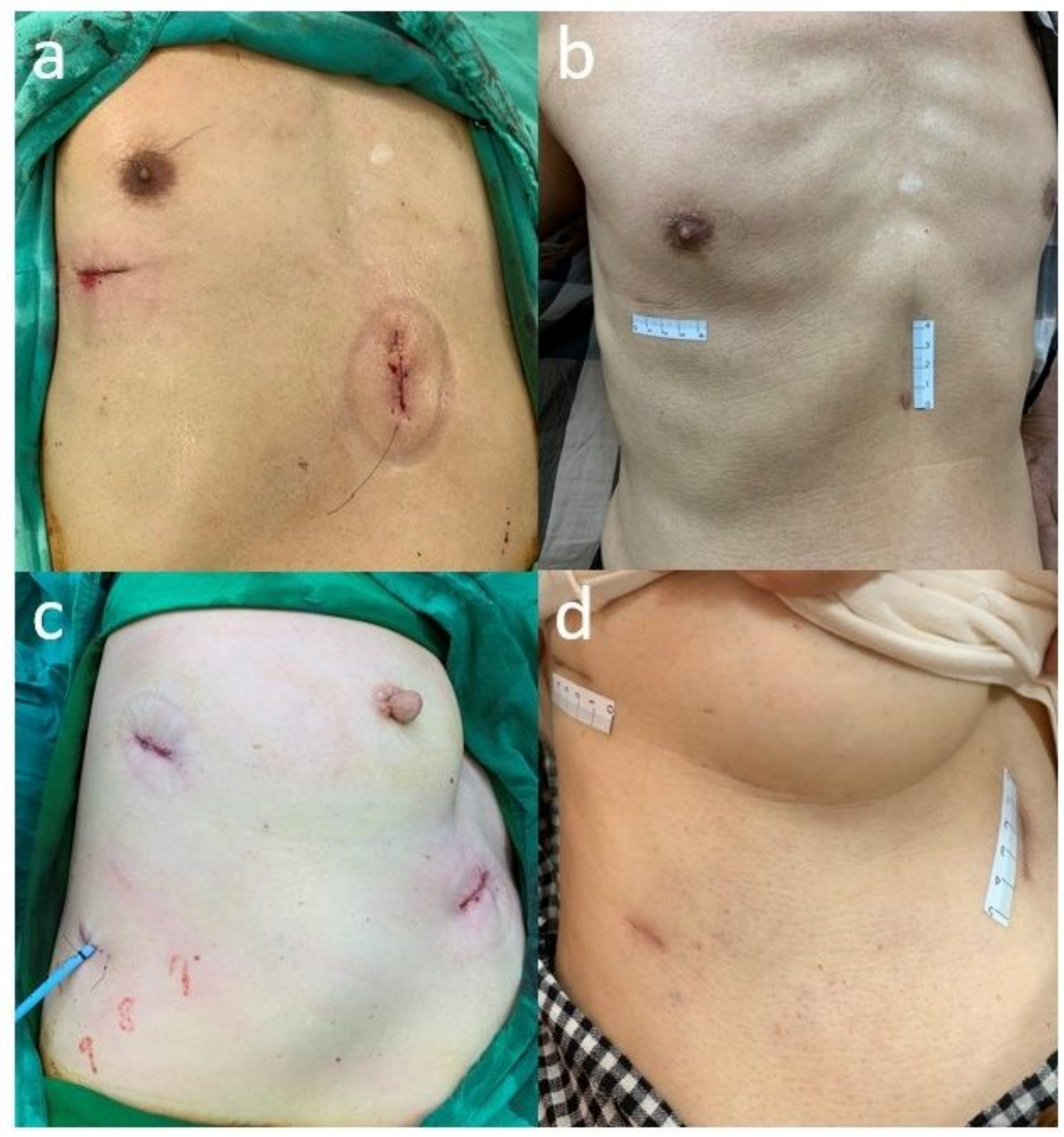

\section{Figure 4}

Patients receiving multiportal subxiphoid and intercostal VATS (a) A patient with anterior mediastinal tumor received two-port VATS resection with equal length of subxiphoid and intercostal incision as well as no postoperative drain insertion. (b)

Postoperative picture of the above-mentioned patient 3 months following surgery. (c) A patient with lung cancer received 3-port VATS right lower lobectomy with equal length of subxiphoid and intercostal incisions with drain-insertion via the small 7th intercostal wound. (d) Postoperative picture of the above-mentioned patient 3 months following surgery. VATS, video-assisted thoracoscopic surgery 


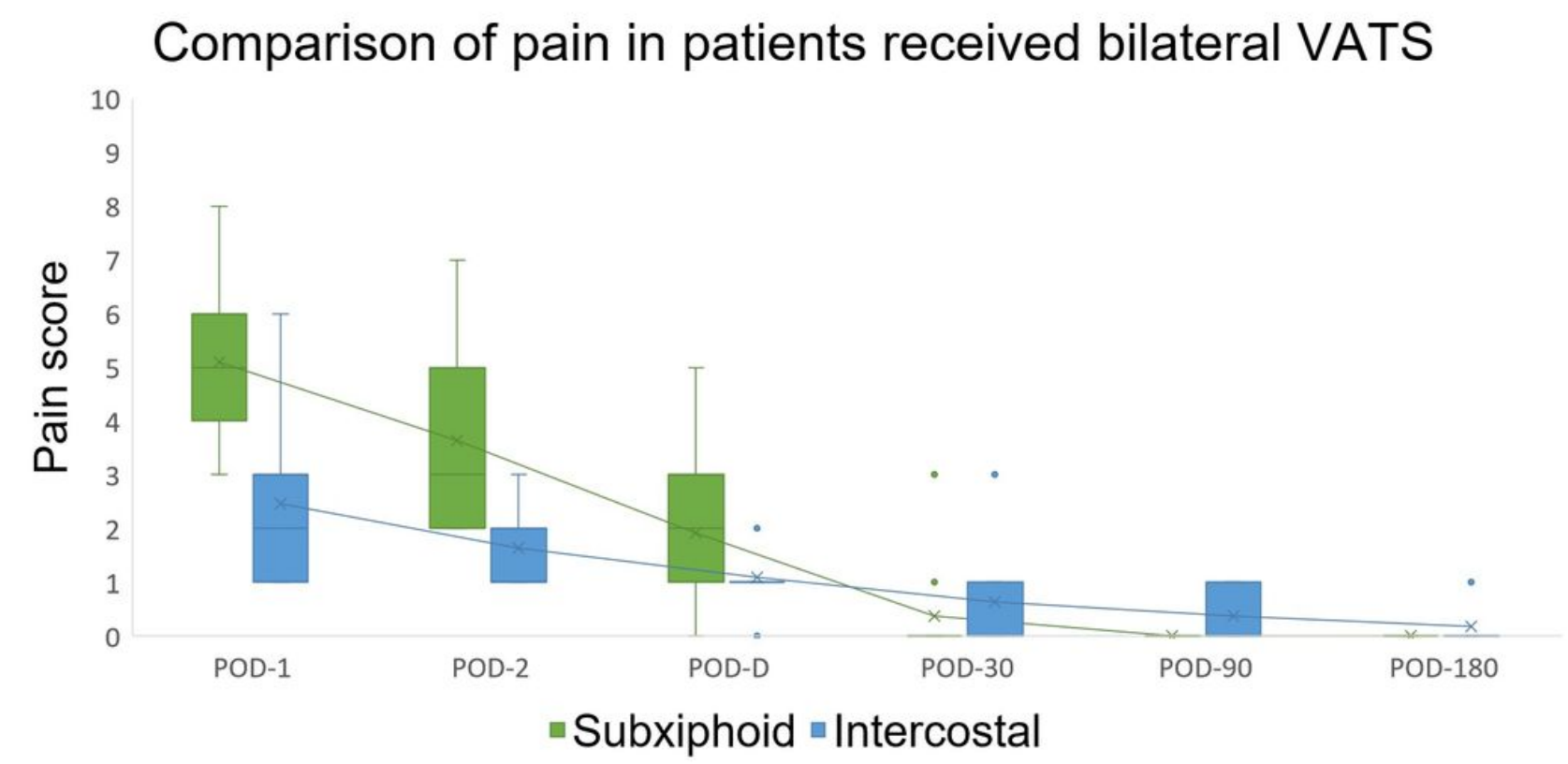

Figure 5

A Box-Whisker plot demonstrating pain scores between subxiphoid and intercostal incisions for the same patient received bilateral uniportal VATS Subxiphoid is shown in green, with intercostal in blue. POD, postoperative day. D, discharge

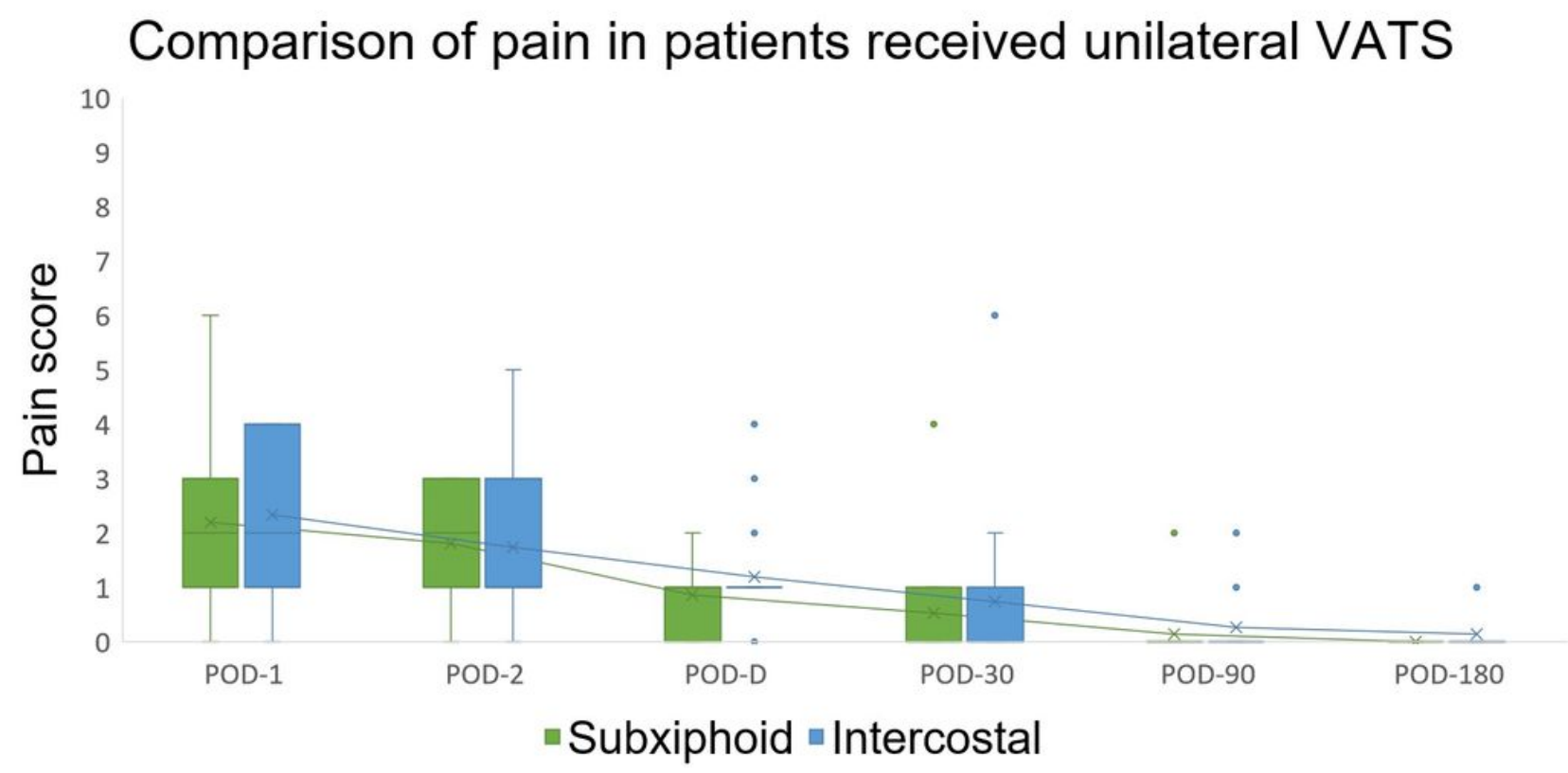

Figure 6 
A Box-Whisker plot demonstrating pain score between subxiphoid and intercostal incisions for the same patient received unilateral multiportal VATS Subxiphoid is shown in green, with intercostal in blue. POD, postoperative day. D, discharge

\section{Supplementary Files}

This is a list of supplementary files associated with this preprint. Click to download.

- SupplementalFig1.tif

- SupplementalFig2.tif

- SupplementalTable1.docx

- SupplementalTable2.docx 